\title{
EFFECT OF SPIN-GLASS PHASE FORMATION ON EXCITON MAGNETIC POLARON IN (Cd,Mn)Te
}

\author{
A.L. Chudnovskiy, B. Rosenow, R. Oppermann, D.R. Yakovlev*, \\ U. ZEHNDER AND W. OSSAU \\ Physikalisches Institut, Universität Würzburg, \\ Am Iubland, 97074, Würzburg, Germany
}

\begin{abstract}
The influence of the spin-glass phase formation of the (Cd,Mn)Te semimagnetic layers on properties of the exciton magnetic polaron were investigated both theoretically and experimentally. The theoretical model offers a consistent description of the temperature dependence of the exciton magnetic polaron energy in the vicinity of spin-glass transition and shows good agreement with the experimental data.
\end{abstract}

PACS numbers: 75.50.Lk, 75.50.Pp

\section{Introduction}

In exciting an exciton in semimagnetic semiconductor ( $\mathrm{Cd}, \mathrm{Mn}) \mathrm{Te}$ the exciton magnetic polaron (EMP) is formed through the strong exchange interaction of the spin of the hole of the exciton with Mn spins in magnetic CdMnTe layer $[1,2]$. Since there are strong experimental evidences that CdMnTe exhibits a spin-glass (SG) phase transition at low temperature Mn $[3,4]$, one can expect the spin-glass phase of the CdMnTe layer to affect the properties of the EMP. Indeed, the measurements of the circular polarization degree of luminescence in $(\mathrm{Cd}, \mathrm{Mn}) \mathrm{Te}$ with Mn content exceeding $20 \%$ show the characteristical cusp in the temperature dependence which is the evidence of a spin-glass transition [5]. The temperatures of these cusps determined in the range of Mn-content from 0.24 up to 0.43 [6] coincide very well with spin-glass transition temperatures $T_{\mathrm{SG}}$ determined from direct magnetization measurements [4]. The temperature dependence of the EMP energy shows no pronounced cusp at $T_{\mathrm{SG}}$ but a plateau at low temperatures [2], which cannot be modelled with the modified Brillouin function (see [7]). Though the plateau occurs at temperatures lower than $T_{\mathrm{SG}}$ we point out that it is due to the spin-glass phase of the magnetic layer. The shift of the temperature of the plateau results from the high local exchange field in EMP. We present a short description of a theory of the interaction of the hole spin of EMP with spin glass.

*On leave from A.F. Ioffe Physico-Technical Institute, Russian Academy of Sciences, 194021, St. Petersburg, Russia. 


\section{Theoretical model}

We suggest the following model of EMP-spin-glass interaction. The magnetic system (CdMnTe layer) consists of $N$ spins, a part $\xi N$ of which constitutes a percolated cluster with random frustrated interaction between spins, hence a spin glass at low temperatures. The rest of the magnetic system is composed of relatively small clusters of antiferromagnetically coupled spins. The spin of the hole of the exciton interacts with a part of $N_{1}=\alpha N$ spins which contains the spins of $\mathrm{SG}$ as well as the spins of antiferromagnetic (AF) clusters (see Fig. 1). Therefore the EMP contains $\alpha \xi N$ spins of SG and $\alpha(1-\xi) N$ spins of AF clusters. Basing on the Villain model of the spin glass in isolating systems of Ileisenberg spins (see [3]) we consider the spin glass as being composed of Ising pseudospins $P_{i}= \pm 1$.

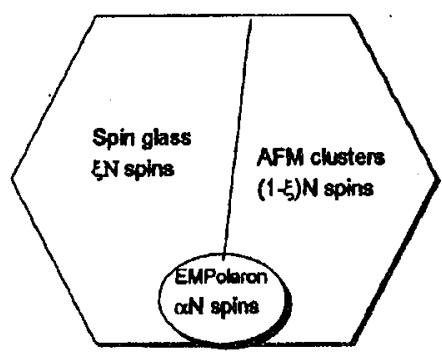

Fig. 1. The theoretical picture of the EMP-SG interaction adopted here.

The Hamiltonian

$$
\begin{aligned}
H= & -\kappa_{\mathrm{SG}} s^{z} \sum_{i \in \mathrm{SGclust}} P_{i}-\kappa_{\mathrm{AF}} s^{z} \sum_{i \in \mathrm{AFclust}} S_{i}^{z} \\
& -\sum_{(i, j) \in \mathrm{SG}} J_{i j} P_{i} P_{j}+\sum_{(i j) \in \mathrm{AF} \text { clust }} J_{\mathrm{AF}} S_{i} S_{j}
\end{aligned}
$$

contains the following terms:

$-\kappa_{\mathrm{SG}} s^{z} \sum_{i \in \mathrm{SG} \text { clust }} P_{i}-$ thic Kondo-like interaction between the spin of the hole of the exciton $s^{z}$ and pseudospins of the cluster of the spin glass;

$-\kappa_{\mathrm{AF}} s^{z} \sum_{i \in \mathrm{AFclust}} S_{i}^{z}-$ the Kondo-like interaction between the spin of the hole of the exciton and spins of the antiferromagnetic clusters;

$-\sum_{(i j) \in \mathrm{SG}} J_{i j} P_{i} P_{j}-$ the random interaction between spins of the spin glass with Gaussian-distributed random $J_{i j}$ and the convention that $(i j)$ counts pairs $i j$ only once and only with $i \neq j$; clusters.

$\sum_{(i j) \in \mathrm{AF} \text { clust }} J_{\mathrm{AF}} \boldsymbol{S}_{i} \boldsymbol{S}_{j}-$ the interaction between spins in antiferromagnetic

With the help of the replica-trick [8] we write the partition function of the system and after the decoupling procedure obtain the following saddle point equations for the spin-glass order parameter $q$, average magnetizations in $S G$ and $A F$ parts of EMP $m_{\mathrm{SG}}$ and $m_{\mathrm{AF}}$ and average spin of the hole $p$ :

$$
q=\int_{G} \mathrm{~d} z \tanh ^{2}\left[\beta\left(J z \sqrt{q}+\frac{\kappa_{\mathrm{SG} p}}{N_{1}}+h_{\mathrm{ext}}\right)\right],
$$




$$
\begin{aligned}
& m_{\mathrm{SG}}=\int_{G} \mathrm{~d} z \tanh \left[\beta\left(J z \sqrt{q}+\frac{\kappa_{\mathrm{SG}} p}{N_{1}}+h_{\mathrm{ext}}\right)\right], \\
& m_{\mathrm{AF}}=S_{\mathrm{eff}} B_{5 / 2}\left(\frac{\frac{\kappa_{\mathrm{AF} p}}{N_{1}}+h_{\mathrm{ext}}}{T+T_{\mathrm{eff}}}\right), \\
& p=\tanh \left[\beta\left(\xi \kappa_{\mathrm{SG}} m_{\mathrm{SG}}+(1-\xi) \kappa_{\mathrm{AF}} m_{\mathrm{AF}}\right)\right] .
\end{aligned}
$$

$$
\int_{G}^{\text {Here }} \mathrm{d} z f(z) \equiv \frac{1}{\sqrt{2 \pi}} \int_{-\infty}^{\infty} \mathrm{d} z \mathrm{e}^{-z^{2} / 2} f(z) .
$$

The antiferromagnetic clusters were treated phenomenologically with the help of the modified Brillouin function (see [7]). The values $\kappa_{\mathrm{SG}} p / N_{1}$ and $\kappa_{\mathrm{AF}} p / N_{1}$ are the exchange fields between the spin of the hole and spins in SG or AF-clusters respectively.

\section{Comparison with experiment}

In order to check the validity of the suggested model we compared its results with the experimental data for the $\mathrm{Cd}_{0.67} \mathrm{Mn}_{0.33} \mathrm{Te}$ epilayer. Temperature dependences of a magnetic-field-induced circular polarization degree of exciton luminescence, which reflects the magnetic susceptibility [9], and of a magnetic polaron energy, determined under selective excitation of localized excitons [2], are presented in Fig. 2. The saddle-point equations of the previous part have been

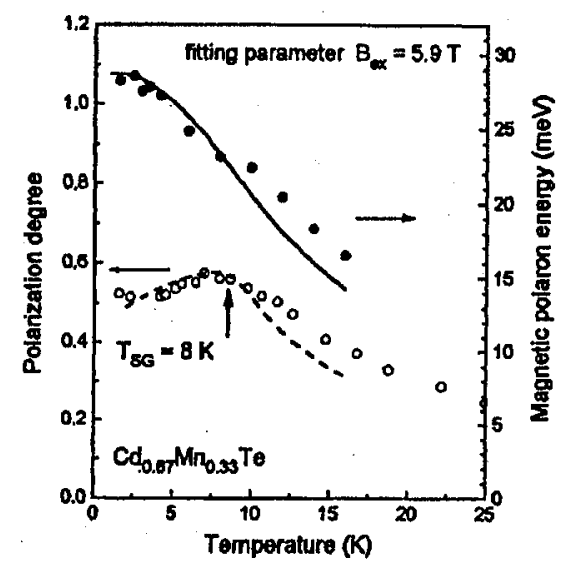

Fig. 2. The temperature dependence of EMP energy (closed circles) and the magnetic-field-induced polarization degree measured at $0.4 T$ (open circles) of a MBE-grown epilayer $\mathrm{Cd}_{0.67} \mathrm{Mn}_{0.33} \mathrm{Te}$. Results of theoretical calculations are shown by solid and dashed lines respectively. 
solved numerically with parameters typical of $\mathrm{Cd}_{0.67} \mathrm{Mn}_{0.33} \mathrm{Te}$ and the temperature dependence of EMP energy has been analysed. The EMP energy was calculated according to the formula

$$
\epsilon=p\left[\xi \kappa_{\mathrm{SG}} m_{\mathrm{SG}}+(1-\xi) \kappa_{\mathrm{AF}} m_{\mathrm{AF}}\right] .
$$

The temperature dependence of EMP energy shows a plateau at temperatures lower than the spin-glass transition temperature in zero magnetic field in accordance with experimental data.

Fixing the value of $J=8 \mathrm{~K}$ (the spin-glass transition temperature for $33 \% \mathrm{Mn}$ [4]) we fitted the experimental curve for the EMP energy (see Fig. 2). The best fit of experimental data shown by solid line gives the value of exchange field in EMP $B_{\mathrm{ex}} \approx \kappa_{\mathrm{SG}} p / N_{1}$ of $5.9 \mathrm{~T}$ which is close to the experimental value of about $5 \mathrm{~T}$ estimated from the exciton Zeeman splitting $[9,6]$. The number of spins in the polaron $N_{1} \approx 80$ which is also in very reasonable agreement with experimental data [9]. The obtained results corroborate the assumption of the spin glass as the reason for the observed plateau in the temperature dependence of EMP energy.

In conclusion, we worked out a theory of interaction of a single spin with a spin glass, which describes the properties of exciton magnetic polaron at low temperatures and is in good agreement with the experimentally measured data. The theory explains the existence of a plateau in the EMP energy at low temperatures. The local exchange magnetic field in the polaron shifts the temperature of SG transition in the polaron to that in bulk which explains why the plateau occurs at a lower temperature than the SG transition temperature.

\section{Acknowledgments}

This work is supported by the Deutsche Forschungsgemeinschaft through the Sonderforschungsbereich 410 .

\section{References}

[1] P.A. Wolff, in: Semiconductors and Semimetals, Vol. 25, Eds. J. Furdyna, J. Kossut, Academic Press, London 1988, p. 413.

[2] G. Mackh, W. Ossau, D.R. Yakovlev, A. Waag, G. Landwehr, R. Hellmann, E.O. Goebel, Phys. Rev. B 49, 10248 (1994).

[3] A. Mauger, J. Villain, Y. Zhou, C. Rigaux, N. Bontemps, J. Ferre, Phys. Rev. B 47, 4587 (1990).

[4] S. Oseroff, P.H. Keesom, in: Semiconductors and Semimetals, Vol. 25, Eds. J. Furdyna, J. Kossut, Academic Press, London 1988, p. 73.

[5] A.V. Kudinov, Yu.G. Kusraev, V.N. Yakimovich, Phys. Solid State 37, 359 (1995).

[6] D.R. Yakovlev, U. Zehnder, W. Ossau, A. Waag, G. Landwehr, T. Wojtowicz, G. Karczewski, J. Kossut, to be published.

[7] J.A. Gaj, R. Planel, G. Fishman, Solid State Commun. 29, 435 (1979).

[8] K. Binder, A.P. Young, Rev. Mod. Phys. 58, 801 (1986).

[9] I.A. Merkulov, D.R. Yakovlev, K.V. Kavokin, G. Mackh, W. Ossau, A. Waag, JETP Lett. 62, 335 (1995). 